\title{
The SF-36 in multiple sclerosis: why basic assumptions must be tested
}

\author{
J Hobart, J Freeman, D Lamping, R Fitzpatrick, A Thompson
}

\begin{abstract}
Objectives-To evaluate, in people with multiple sclerosis, two psychometric assumptions that must be satisfied for valid use of the medical outcomes study 36-item short form health survey (SF-36): the data are of high quality and, it is legitimate to generate scores for eight scales and two summary measures using the standard algorithms.
\end{abstract}

Methods-SF-36 data from 438 people representing the full range of multiple sclerosis were examined (mean age 48; $70 \%$ women). Data quality (per cent missing data and computable scale and summary scores) were determined, six scaling criteria were tested to determine the legitimacy of generating the eight SF-36 scale scores using Likert's method of summed ratings, and two scaling criteria were tested to determine the appropriateness of the standard SF-36 algorithms for weighting scale scores to generate two summary measures.

Results-Data quality was excellent except in the most disabled subgroup where missing responses reached a maximum of $16.5 \%$ and summary scores could only be computed for $72 \%$. There was clear support for the generation of SF-36 scale scores. Item response distributions were symmetric, item mean scores and variances were equivalent, corrected itemtotal correlations were high (range 0.460.85 ) and similar, and definite scaling success rates exceeded $96 \%$. Nevertheless, there were notable floor or ceiling effects in four of the eight scales. Assumptions for generating two SF-36 summary measures were only partially satisfied. Although principal components analysis suggested a two component model, these components explained less than $60 \%$ of the total variance in SF-36 scales, and less than $75 \%$ of the variance in five of the eight scales. Moreover, scale to component correlations did not support the use of scale weights derived from United States population data.

Conclusions-When using the SF-36 as a health measure in multiple sclerosis summary scores should be reported with caution.

(F Neurol Neurosurg Psychiatry 2001;71:363-370)

Keywords: SF-36; tests of scaling assumptions; health measurement; multiple sclerosis

Dr J Hobar

J.Hobart@ion.ucl.ac.uk

Received 29 December 2000 and in final form

26 April 2001

Accepted 30 April 2001
Changes in health policy have underlined the importance of measuring outcomes that are relevant to patients. This has resulted in the increased use of generic, patient reported health status measures. However, the use of these measures assumes that they satisfy minimum psychometric requirements across diverse clinical populations. ${ }^{12}$ This often untested assumption is the subject of this article.

The medical outcomes study 36 item short form health survey (SF-36) is one of the most widely used patient reported health status measures. ${ }^{3}$ It is recommended for use in health policy evaluations, general population surveys, clinical research, and clinical practice. ${ }^{4}$ In neurology, the SF-36 has been used in stroke, ${ }^{5}$ motor neuron disease, ${ }^{6}$ Parkinson's disease, ${ }^{7}$ epilepsy, ${ }^{8}$ headache, ${ }^{9}$ and multiple sclerosis. ${ }^{10-13}$ Moreover, it has often been used as a validating instrument in the psychometric evaluation of new measures. ${ }^{14-19}$

The SF-36 generates two types of scores (fig 1). To generate scores for the eight SF-36 scales, items are summed without weighting or standardisation. To generate scores for the two SF-36 summary measures, scale scores are weighted and combined. Although the eight scales provide a more comprehensive profile of health status, the two summary measures have features that make them more advantageous for clinical trials. These include better measurement precision, smaller confidence intervals, the elimination of floor and ceiling effects, simpler analysis by reducing the number of statistical tests required and avoiding the problem of multiple testing, and superior (theoretically) responsiveness. ${ }^{20} 21$ Summary measures are also more easily interpreted as their scores are directly related to scores for the general United States population, which have been transformed to a mean of 50 and an SD of 10 .

Despite the widespread use of the SF-36 in multiple sclerosis, and the demonstration of some of its psychometric properties, ${ }^{1522} 23$ no study has comprehensively examined two fundamental prerequisites for rigorous measurement: indicators of data quality and tests of scaling assumptions. Indicators of data quality, such as item non-response and missing scale and summary scores, determine the legitimacy of using an instrument. They reflect respondents' understanding and acceptance of a measure and help to identify items that may be irrelevant, confusing, or upsetting to patients. ${ }^{1}$ When there is a large amount of missing data, scores for scales and summary measures cannot be reliably estimated. Tests of scaling assumptions determine whether it is legitimate to generate scores for an instrument using the algorithms proposed by the developers. As data quality and psychometric properties are sample 
dependent, ${ }^{1}{ }^{24}$ the performance of a measure in a specific application is more important than its performance generally. ${ }^{1}$ This study examines data quality and tests the scaling assumptions for the SF-36 in a sample of people with multiple sclerosis.

\section{Methods}

PARTICIPANTS, RECRUITMENT, AND DATA

COLLECTION

Data were analyzed from two ethically approved studies conducted at the National Hospital for Neurology and Neurosurgery (NHNN) in London. Participants in the first study were adults with clinically definite multiple sclerosis attending the NHNN. ${ }^{25}$ Full details of the sampling and the stratification process are described elsewhere. ${ }^{23}$ Briefly, 150 consecutive attenders were recruited from three different sources: a weekly outpatient clinic, an inpatient neurological rehabilitation unit, and admissions under one consultant (AT). People in this hospital based sample were excluded if they had cognitive impairment precluding reliable completion of questionnaires (subjective judgement of JF), other comorbid disabling disorders, or were not English speaking. A stratification process was used to ensure an even spread of disease severity: all patients were examined by a neurology registrar and classified according to the Kurtzke expanded disability status scale $\left(\right.$ EDSS $\left.^{26}\right)$ as either mild (EDSS $\leqslant 4.5$ ), moderate (EDSS 5.0-6.5), or severe (EDSS $\geqslant 7.0$ ). Consecutive admissions were recruited until there were 50 patients in each category. The SF-36 was administered by a single investigator (JF) in strict accordance with the developers' recommendations. ${ }^{3}$

The second study was a postal survey of 500 randomly selected, geographically stratified members of the multiple sclerosis Society of Great Britain and Northern Ireland. This was part of a larger study developing a patient based outcome measure for multiple sclerosis. ${ }^{27}$ The SF-36 was administered in a booklet along with three other health measures and

\section{Scale}

Summary measure

\begin{tabular}{|ll|}
\hline $3 \mathrm{a}$ & Limited doing vigorous activities \\
$3 \mathrm{~b}$ & Limited doing moderate activities \\
$3 \mathrm{c}$ & Limited lifting or carrying groceries \\
$3 \mathrm{~d}$ & Limited climbing several flights of stair \\
$3 \mathrm{e}$ & Limited climbing one flight of stairs \\
$3 \mathrm{f}$ & Limited bending, kneeling, stooping \\
$3 \mathrm{~g}$ & Limited walking more than a mile \\
$3 \mathrm{~h}$ & Limited walking half a mile \\
$3 \mathrm{i}$ & Limited walking one hundred yards \\
$3 \mathrm{j}$ & Limited bathing or dressing yourself \\
\hline
\end{tabular}

4a Cut down amount of time spent on work

$4 \mathrm{~b}$ Accomplished less than would like

4c Limited in the kind of work

$4 d \quad$ Difficulty performing the work

$7 \quad$ Pain - magnitude

8 Pain - interference with work

1 Overall rating of general health

11a I seem to get sick easier than others

$11 \mathrm{~b} \mathrm{I} \mathrm{am}$ as healthy as anyone I know

11c I expect my health to get worse

11d My health is excellent

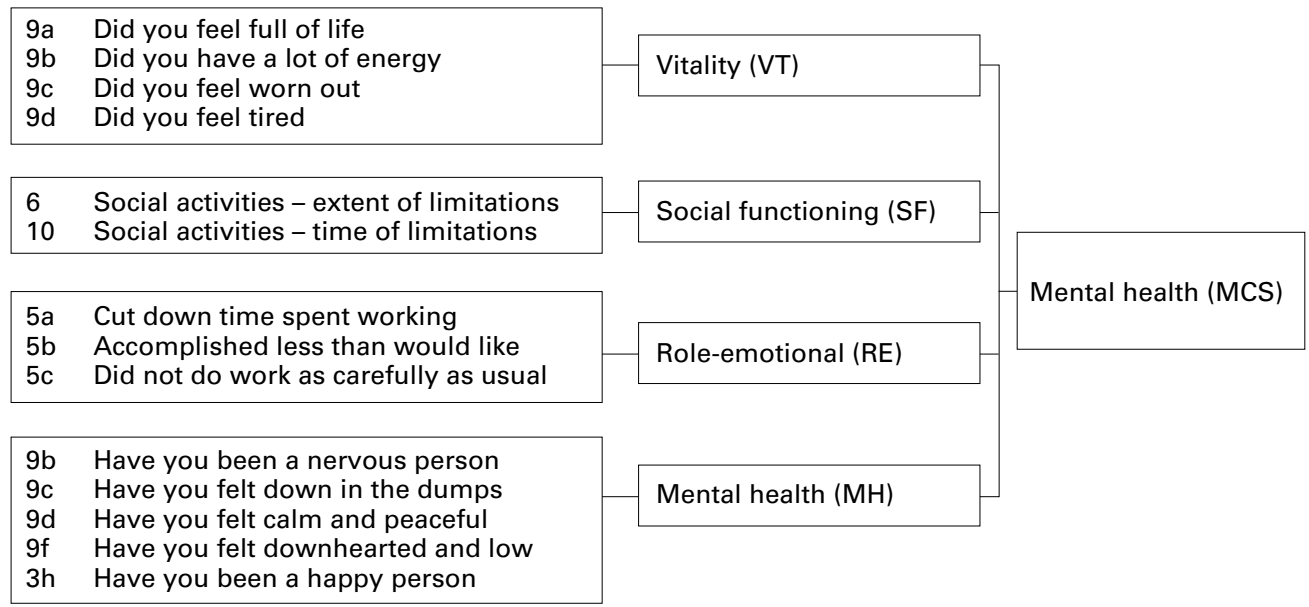

Bodily pain (BP)

General health $(\mathrm{GH})$

Role-physical (RF)

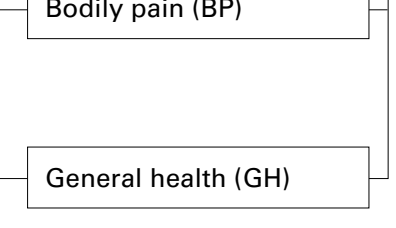

\section{(1)}
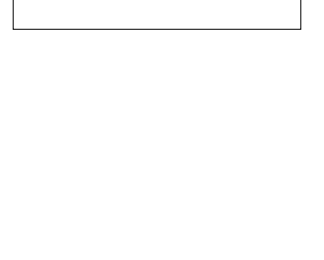

Physical functioning (PF)

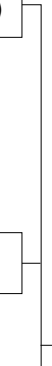

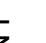


demographic questions. Non-responders were sent reminders at 3 and 5 weeks.

THE MEASUREMENT MODEL OF THE SF-36

The measurement model of the SF-36 (fig 1) hypothesises that 35 of the 36 items are grouped into eight multi-item scales (physical functioning $(\mathrm{PF})$, role limitations physical (RP), bodily pain (BP), general health perceptions $(\mathrm{GH})$, energy/vitality (VT), social functioning (SF), role limitations emotional (RE), and mental health $(\mathrm{MH})$ that are aggregated into two summary measures (physical component (PCS), mental component (MCS)). The remaining item is not used in scoring.

Different processes are used to generate scores for scales and summary measures. Likert's method of summated ratings is used for scales. $^{28}$ That is, item responses are summed without weighting or standardisation. Before this is undertaken, two items are recalibrated and the scoring of nine items is reversed so that high scores always indicate better health. ${ }^{3}$ Finally, as the eight scales have different ranges they are transformed to have a common range of 0 (worst health) to 100 (best health) using the formula provided by Stewart and Ware. ${ }^{29}$

Scores for the two summary measures are generated in three stages. Firstly, scale scores are standardised ( $z$ score transformation) by subtracting the United States population mean for that scale and dividing the difference by the United States population standard deviation for that scale. Next, $z$ scores are multiplied by their respective factor score coefficients, derived from United States population data, and summed. Finally, these aggregated scores are standardised using a linear $T$ score transformation to have a mean of 50 and SD 10, in the general United States population. ${ }^{20}$

The results of factor analytical studies led to the discovery of the two SF-36 summary measures. Principal components analysis of intercorrelations among SF-36 scales from several studies consistently extracted two components, with similar scale to component correlations, which accounted for most of the total SF-36 variance and variance in each of the individual SF-36 scales. ${ }^{20}$ These findings indicated that two summary measures could be generated without substantial loss of information. The two components were interpreted as measures of physical and mental health because the scales correlating highest with them were $\mathrm{PF}$ and $\mathrm{RP}$, and $\mathrm{MH}$ and $\mathrm{RE}$, respectively.

ANALYSIS PLANS

All analyses were undertaken in the hospital and postal samples separately, and in the pooled sample.

\section{Are the data high quality?}

This was determined by calculating the percentage of missing data for items, and the percentage of scale and summary scores that could be computed. A scale score can be calculated provided that $50 \%$ or more of the items are completed. Missing items are replaced with a person specific mean score, the average score across completed items for that respondent. ${ }^{3} 30$ By contrast, scores can only be calculated for summary measures when all eight scale scores are available. ${ }^{20}$ The impact of disease severity on data quality was also examined. In the hospital based sample, participants were categorised as mild, moderate, or severe by their EDSS score. In the postal survey, participants were categorised by their indoor mobility as walking unaided, walking with an aid, or wheelchair dependent.

Is it legitimate to report SF-36 scale scores in multiple sclerosis?

Six scaling assumptions must be satisfied for SF-36 scale scores to be generated using the item groups proposed by the developers and Likert's method of summated ratings.

(1) Items in each scale must be roughly parallel (that is, measure at the same point on the scale and have similar variances), otherwise they do not contribute equally to the variance of the total score and must be standardised before combination. ${ }^{28}$ This criterion is evaluated by examining the symmetry of item response distributions and the equivalence of item means scores and SD. ${ }^{1}$

(2) Items in each scale must measure a common underlying construct, otherwise it is not appropriate to combine them to generate a total score. ${ }^{28}$ This criterion is evaluated by examining the correlation between each item and the total score computed from the remaining items in that scale (corrected item-total correlation). A range of recommended minimum values has been recommended: $0.20,{ }^{31}$ $0.30,^{32}$ and $0.40 .^{1}$

(3) Items in each scale should contain a similar proportion of information concerning the construct being measured, otherwise they should be given different weights. ${ }^{28}$ This criterion is determined by examining the equivalence of corrected item-total correlations. Recently, Ware et al have stated that this criterion can be considered satisfied when values exceed 0.30 , even if they vary. ${ }^{33}$

(4) Items must be correctly grouped into scales. That is, items must correlate substantially higher with the construct they are purported to measure than with the other constructs measured by the instrument. ${ }^{34}$ This criterion is considered satisfied when item-own scale correlations (corrected for overlap) exceed item-other scale correlations by at least two standard errors $(2 \times 1 / \sqrt{n})$.

(5) Scales must generate reliable estimates, otherwise their scores cannot be confidently interpreted. This criterion is satisfied when Cronbach's $\alpha$ coefficients $^{35}$ for each scale exceed $0.70^{36}$ or $0.80 . .^{32}$

(6) Scales must demonstrate that they measure distinct constructs, otherwise interpretation of their scores is confounded. This criterion is satisfied when the correlations among scales are substantially less than their respective reliability estimates. ${ }^{33}$ 
Is it legitimate to report SF-36 summary scores in multiple sclerosis?

Two scaling assumptions must be satisfied for SF-36 PCS and MCS scores to be generated using the developers' algorithm. ${ }^{3} 32$

(1) Principal components analysis, with orthogonal (varimax) rotation of extracted components, of the correlations among the eight SF-36 scales should support a two dimensional model of health that explains about $80 \%-85 \%$ of the total reliable variance in the SF-36 scales and at least $75 \%$ of the reliable variance in each of the eight SF-36 scales. ${ }^{3} 20$

(2) The magnitude and pattern of correlations between the eight SF-36 scales and the two rotated components should support their interpretation as measures of physical and mental health, and be consistent with other studies. That is, the PCS measure should correlate strongly $(>0.70)$ with the $\mathrm{PF}, \mathrm{RP}$, and $\mathrm{BP}$ scales, and weakly $(<0.30)$ with the $\mathrm{MH}$ and $\mathrm{RE}$ scales, and vice versa for the mental MCS measure. $^{3}$

These two scaling assumptions were examined by undertaking a scale level principal components analysis with varimax rotation. Eigenvalues $^{37}$ and the scree plot $^{38}$ were examined to determine the optimum number of components to rotate.

\section{Results}

SAMPLE CHARACTERISTICS

In the hospital based study, 150 people were recruited and one person withdrew. In the postal survey, a total of 409 booklets (82\%) were returned; 121 questionnaires were returned blank, of these 84 were considered ineligible to participate in the study (changed address; did not have multiple sclerosis; deceased). Therefore, the response rate was $69 \%$ (409-121/500-84). The characteristics of patients in the two samples were similar, a wide range of ages and disease duration was included, and the broad categories of disability were evenly represented (table 1).

ARE THE DATA HIGH QUALITY ?

For the hospital based sample there were no missing data, both types of SF-36 scores could be estimated for all participants, and results were not influenced by disease severity (table $2)$. In the postal survey, data quality was excellent in participants who reported that they

Table 1 Characteristics of samples

\begin{tabular}{|c|c|c|}
\hline & \multicolumn{2}{|l|}{ Sample } \\
\hline & Hospital & Postal \\
\hline No of patients & 149 & 288 \\
\hline Women (\%) & 68 & 72 \\
\hline Age (y) (mean (SD; range)) & $45(11 ; 24-78)$ & $52(12 ; 21-80)$ \\
\hline Years since diagnosis (mean (SD; range)) & $10(8 ; 0.1-38)$ & $14(10 ; 1-51)$ \\
\hline Years since first symptoms (mean (SD; range)) & $15(9 ; 0.6-43)$ & $19(11 ; 1-59)$ \\
\hline \multicolumn{3}{|l|}{ Disability level (\%) } \\
\hline Mild (EDSS $\leqslant 4.5)$ & 32 & N/A \\
\hline Moderate (EDSS 5.0 to 6.5 ) & 34 & N/A \\
\hline Severe (EDSS $\geqslant 7.0)$ & 34 & N/A \\
\hline \multicolumn{3}{|l|}{ Indoor mobility $\% \star$} \\
\hline Walk unaided & N/A & 33 \\
\hline Walk aided & N/A & 38 \\
\hline Use a wheel chair & N/A & 29 \\
\hline
\end{tabular}

*This question was completed by $94.8 \%(n=273)$ of the sample.
Table 2 Per cent item non-response and computable scale and summary scores

\begin{tabular}{|c|c|c|c|c|}
\hline \multirow[b]{2}{*}{ Sample } & \multirow{2}{*}{$\begin{array}{l}\text { No of } \\
\text { patients }\end{array}$} & \multirow{2}{*}{$\begin{array}{l}\text { Item } \\
\text { non-response } \\
(\%)\end{array}$} & \multicolumn{2}{|c|}{ Computable scores (\%) } \\
\hline & & & Scales & $P C S / M C S$ \\
\hline Pooled & 437 & $0.9-5.7$ & $95.7-100$ & 90.9 \\
\hline Hospital & 149 & 0 & 100 & 100 \\
\hline Postal survey & 288 & $1.0-7.3$ & $92.4-100$ & 86.5 \\
\hline $\begin{array}{l}\text { Walk } \\
\text { unaided }\end{array}$ & 90 & $0-4.5$ & $96.7-100$ & 95.5 \\
\hline Walk aided & 104 & $1.0-5.8$ & $94.2-100$ & 91.3 \\
\hline Wheel chair & 79 & $1.3-16.5$ & $84.8-100$ & 72.2 \\
\hline
\end{tabular}

walked unaided or with an aid. In wheelchair dependent participants, the proportion of missing data reached a maximum of $16.5 \%$ for two items (questions $3 i, 3 j$ ) and, although scale scores could be computed for the vast majority of people, scores for summary measures could only be computed in $72 \%$ (all eight scale scores must be present for summary scores to be computed).

IS IT LEGITIMATE TO GENERATE SF-36 SCALE SCORES IN MULTIPLE SCLEROSIS ?

Results for the pooled sample are reported, but independent analyses of the hospital and postal samples yielded similar results. Although all response options were endorsed for each item, item response-option frequency distributions were quite symmetric for only five of the eight scales (BP, GH, VT, SF, RE). Response distributions were skewed towards less favourable health states (low scores) for the PF and RP scales, and skewed towards more favourable health states for the MH scale. Nevertheless, items within each scale had similar mean scores and standard deviations indicating that they were roughly parallel.

All item-own scale correlations, corrected for overlap, exceeded 0.40 indicating that the items in each scale measured a common underlying construct, and that the criterion of Ware et al of equivalence of item total correlations was satisfied. Only two item-own scale correlations did not exceed item-other scale correlations by more than two standard errors. Item 9a ("How much of the time did you feel full of life") correlated similarly with the VT and SF scales (0.61 and 0.54 respectively), and item 9b ("How much of the time have you been a nervous person") correlated similarly with the $\mathrm{MH}$ and RE scales ( 0.46 and 0.36 respectively). Therefore, these two items had a limited ability to discriminate between two constructs that are hypothesised to be distinct.

$\alpha$ Coefficients for all SF-36 scales exceeded 0.80 indicating that all scales generated reliable scores (table 3). Intercorrelations among scales (range 0.18 to 0.57 ) were substantially below their respective $\alpha$ values, indicating that they were measuring eight related but distinct constructs.

Scores for the eight SF-36 scales spanned the entire scale ranges and, therefore, demonstrated good variability. Scores for the PF and RP scales were positively skewed (skewness 1.13 and 1.32 respectively) indicating that respondents tended to be more physically disabled. Scores for the other six scales were more evenly distributed 
Table 3 Intercorrelations among SF-36 scales in the pooled sample ( $n=415$ to 437)

\begin{tabular}{|c|c|c|c|c|c|c|c|c|}
\hline \multirow{2}{*}{$\begin{array}{l}\text { SF-36 } \\
\text { scale }^{\star}\end{array}$} & \multicolumn{8}{|c|}{ SF-36 scale } \\
\hline & $P F$ & $R P$ & $B P$ & $G H$ & $V T$ & $S F$ & $R E$ & $M H$ \\
\hline PF & $(0.94)$ & & & & & & & \\
\hline $\mathrm{RP}$ & 0.49 & $(0.87)$ & & & & & & \\
\hline $\mathrm{BP}$ & 0.30 & 0.28 & $(0.92)$ & & & & & \\
\hline $\mathrm{GH}$ & 0.37 & 0.37 & 0.34 & $(0.81)$ & & & & \\
\hline VT & 0.34 & 0.43 & 0.35 & 0.48 & $(0.82)$ & & & \\
\hline SF & 0.45 & 0.48 & 0.34 & 0.48 & 0.49 & $(0.83)$ & & \\
\hline RE & 0.18 & 0.45 & 0.28 & 0.28 & 0.35 & 0.44 & $(0.89)$ & \\
\hline $\mathrm{MH}$ & 0.23 & 0.33 & 0.34 & 0.44 & 0.54 & 0.51 & 0.57 & $(0.83)$ \\
\hline
\end{tabular}

^Abbreviations in text.

$\alpha$ Coefficients in parentheses.

Table 4 Correlations between SF-36 scales and rotated components

\begin{tabular}{|c|c|c|c|c|c|c|c|}
\hline \multirow[b]{2}{*}{ SF-36 scalet } & \multirow[b]{2}{*}{ Factor $\neq$} & \multicolumn{3}{|c|}{$M S(n=398)$} & \multicolumn{3}{|c|}{ US population $(n=2474)$ * } \\
\hline & & PCS & $M C S$ & $h^{2} / r_{t} \delta$ & PCS & $M C S$ & $h^{2} / r_{t t}$ \\
\hline PF & $\mathrm{P}$ & 0.88 & 0.05 & 0.82 & 0.85 & 0.12 & 0.78 \\
\hline $\mathrm{RP}$ & $\mathrm{P}$ & 0.69 & 0.30 & 0.67 & 0.81 & 0.27 & 0.82 \\
\hline $\mathrm{BP}$ & $\mathrm{P}$ & 0.48 & 0.31 & 0.35 & 0.76 & 0.28 & 0.72 \\
\hline GH & $\mathrm{P}>\mathrm{M}$ & 0.59 & 0.39 & 0.60 & 0.69 & 0.37 & 0.78 \\
\hline VT & $M>P$ & 0.47 & 0.57 & 0.67 & 0.47 & 0.64 & 0.75 \\
\hline SF & M & 0.60 & 0.51 & 0.74 & 0.42 & 0.67 & 0.92 \\
\hline RE & M & 0.13 & 0.81 & 0.75 & 0.17 & 0.78 & 0.78 \\
\hline $\mathrm{MH}$ & M & 0.20 & 0.86 & 0.91 & 0.17 & 0.87 & 0.92 \\
\hline Eigenvalue & & 3.78 & 0.996 & & $>1.0$ & $>1.0$ & \\
\hline Variance & & 47.3 & 12.5 & & NRף & NR & \\
\hline Total variance ${ }^{\star \star}$ & & 59.7 & & & 81.5 & & \\
\hline
\end{tabular}

$\star$ From Ware et al 1994.

†Abbreviations in text.

$\ddagger$ Hypothesised factor content: $\mathrm{P}=$ physical factor content; $\mathrm{M}=$ mental factor content.

$§$ Total reliable variance in each SF-36 scale explained by the two principal components. $\mathrm{h}^{2}=$ sum of squared factor loadings for each scale; $r_{t}=\alpha$ coefficient for each scale.

$\uparrow$ Not reported.

$\star \star$ Per cent of total reliable variance in all SF-36 scales explained by the two principal components.

(skewness -0.50 to +0.40 ). There were notable floor or ceiling effects for four scales: RP (floor $61.6 \%$ ); RE (floor $37.3 \%$, ceiling $41.4 \%$ ); $\mathrm{PF}$ (floor 23.4\%); BP (ceiling 21.1\%).

IS IT LEGITIMATE TO GENERATE SF-36 SUMMARY SCORES IN MULTIPLE SCLEROSIS?

Results for the pooled sample are reported, but independent analyses of the hospital and postal samples yielded similar results. Principal components analysis of intercorrelations among SF-36 scales extracted only one component with an Eigenvalue greater than unity. Nevertheless, the Eigenvalue of the second component (0.996) was essentially unity and examination of the scree plot supported the hypothesis that a two dimensional model of health underpins the SF-36 in multiple sclerosis. However, these two components explained less than $60 \%$ of the total reliable variance in all SF-36 scales, and less than $75 \%$ of the reliable variable in five of the eight scales (table 4), indicating that a substantial amount of information from SF-36 scales is lost when summary measures are reported in multiple sclerosis. In addition, the magnitude and pattern of scale to component correlations in multiple sclerosis differ from the United States general population, indicating that the scale weights used to generate scores for the summary measures are not entirely applicable to people with multiple sclerosis (table 4, fig 2).

\section{Discussion}

This study has comprehensively examined the basic assumptions underpinning the use and scoring of the SF-36 in people with multiple
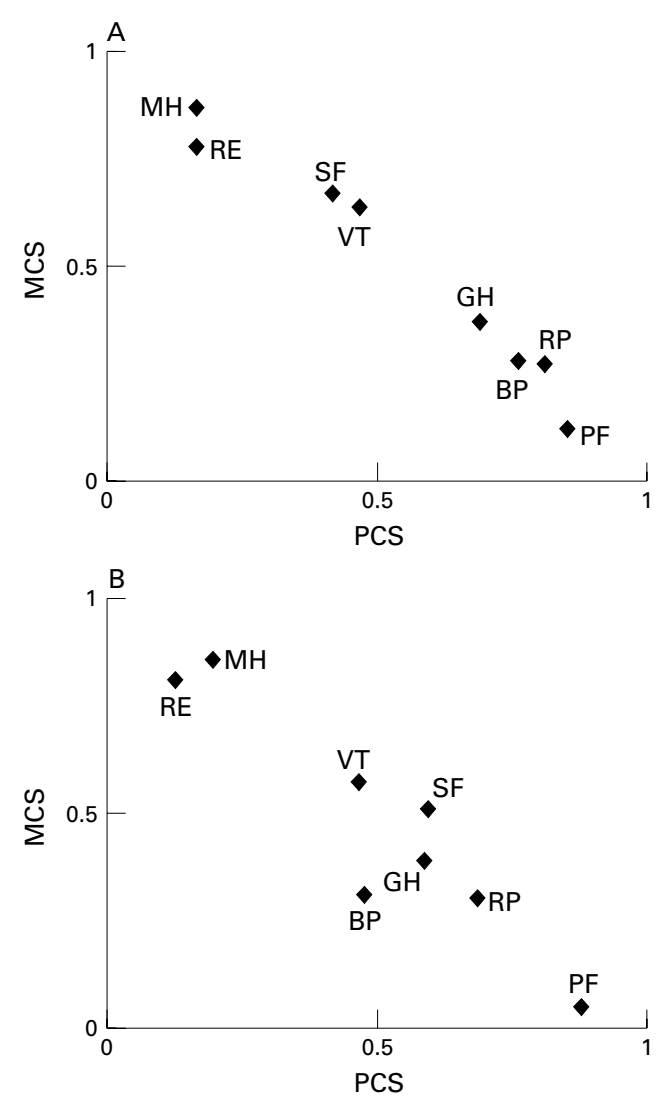

Figure 2 Plot of the SF-36 scale to component correlations. (A) SF-36 in the United States population $(n=2.474)$. Prepared from data reported by Ware et al. ${ }^{20}$ (B) $S F-36$ in multiple sclerosis $(n=438)$.

sclerosis. High levels of data completeness indicate that the SF-36 is acceptable to patients in both hospital and community settings. However, data quality is somewhat compromised when the SF-36 is administered by postal survey to more disabled people. This finding probably represents physical limitations (for example, impaired vision and writing) rather than poor acceptance and understanding of the instrument because data quality in the more disabled hospital based subsample, where the instrument can be administered by interview if required, is excellent.

Results indicate that when using the SF-36 in multiple sclerosis, scale scores can be generated using Likert's method of summed ratings. All scaling assumptions were fully satisfied except item discriminant validity. There were two instances where items failed to achieve definite scaling successes. This is a minor problem because the other items in each scale fully satisfy the criterion and, therefore, anchor the construct being measured. ${ }^{33}$ More problematic are the floor and ceiling effects demonstrated for four scales. These exceed the recommended maximum of $15 \%,{ }^{39}$ and represent subsamples of people for whom changes in health status may be underestimated or not detected by the SF-36.

The most important finding of this study is that scores for the two SF-36 summary measures should be reported with caution. Although PCA supports a two dimensional model of health, this model does not explain as 
much of the variance in SF-36 scales as required and the pattern of scale to component correlations is not entirely consistent with findings in other clinical populations. ${ }^{20}{ }^{40} \mathrm{In}$ particular, the BP scale correlates only moderately with the physical component (0.48), and the SF scale correlates only moderately with the mental component $(0.51)$ and similarly with the physical component (0.60). Although small departures from predicted results are to be expected, and have been reported, ${ }^{2}$ these findings in multiple sclerosis seem to be more significant. The finding that the two components explain less than $60 \%$ of the variance in SF-36 scales suggests that even multiple sclerosis specific algorithms for summary scores, based on principal components analysis with orthogonal rotation, may not be feasible.

Other authors have raised concerns about the SF-36 summary scores because the weightings are based on factor score coefficients generated by orthogonal factor rotations. ${ }^{41-43}$ Simon et al argue that physical and mental health are related and, therefore, orthogonal rotations which assume factors are uncorrelated could generate misleading results. They support their argument with empirical evidence in primary care patients. ${ }^{41}$ Norvedt et al show that the SF-36 MCS underestimates the mental heath impact of multiple sclerosis, ${ }^{43}$ and that the RAND-36 physical and mental summary scores, ${ }^{42}$ the weightings of which are generated by oblique factor rotations (which assume the factors are correlated), provide mental health scores that are more consistent with multiple sclerosis.

The results of our study, however, suggest that the problem may be more fundamental than the method of factor rotation. Factor analysis is a data reduction technique that analyses the relations among a group of variables and identifies clusters of variables that are empirically distinct. Table 3 and figure 2 do not show distinct clusters among SF-36 scales. Indeed, correlations among the four scales hypothesised to correlate highest with the physical health component (PF, RP, BP, and $\mathrm{GH}$ ) are notably lower (range 0.28 to 0.49 ; mean 0.36 ) than those reported by others (range 0.52 to 0.65 ; mean $0.57^{20}$ ) and similar to those between physical and mental scales (range 0.18 to 0.48 ; mean 0.37 ).

In view of the comments of Simon et al, and others who raise concerns about the use of principal components analysis or orthogonal factor rotations in health measurement, ${ }^{44}$ we repeated the factor analytical studies using different methods of extraction (principal axis, maximum likelihood, unweighted and generalised least squares) and oblique (promax) rotation. All methods of factor analysis generated similar results. Most scales (four or more) did not load uniquely on only one factor and had relatively high loadings $(>0.50)$ on both factors. Furthermore, no clear factor solutions were generated. These findings are clinically sound. For example, fatigue can impact significantly on both physical and mental functioning and roles. Although our failure to replicate the SF-36 factor structure may represent cultural differences (United Kingdom versus United States), evidence suggests that this is not the case. ${ }^{45}$

Although this is a relatively small study the generalisability of these results is supported by two facts. Firstly, when tests of scaling assumptions underpinning the generation of SF-36 scale and summary scores were undertaken for the hospital and postal samples separately, similar findings were demonstrated. Secondly, SF-36 scale score distributions, floor and ceiling effects, and $\alpha$ coefficients reported in this study reproduce the findings of others. ${ }^{1522}$ Nevertheless, further studies are required to establish the replicability of these results and the interpretation of these findings. It is also important to note that this study has only considered data quality and scaling assumptions, criteria that should be satisfied before more detailed psychometric evaluations are undertaken. More extensive evaluations of SF-36 scales in people with multiple sclerosis are required to determine the extent to which they are valid and responsive indicators of the health constructs that they purport to measure.

This study did not consider the content validity of the SF-36 in multiple sclerosis. That is, to what extent do the items of the SF-36 compare with issues volunteered as important by patients in open ended interviews. Generic measures are designed to assess health domains thought to be universally relevant. Therefore, they have the advantage of enabling comparisons across diseases and interventions, but the disadvantage of failing to reflect domains and aspects of health that are disease unique. This may influence the potential of generic measures to detect change. These arguments underpin the development of disease specific health measures, and the use of both types of measures in studies. ${ }^{27}$

A limitation of our study was that we used an arbitrary criterion, the subjective judgment of one of the authors, to determine whether people in the hospital based sample had significant cognitive impairment precluding reliable completion of questionnaires. It would have been preferable to have had cognitive function formally evaluated, and a predefined empirically based cut off level for inclusion or exclusion. Unfortunately, such criteria do not exist as the relation between cognitive impairment and ability to complete self report questionnaires in a reliable and valid manner has not been formally evaluated. This is an important area for future research in patient based outcome measurement of neurological disorders associated with cognitive impairment.

The results of this study have implications for clinical trials in multiple sclerosis. Some new and expensive treatments are now available that have been shown to significantly affect the natural history of multiple sclerosis in terms of brain MRI and relapse rate but not impact on disability progression. As the disability measure used in all of these studies, the EDSS, has poor ability to distinguish between groups and individual patients and limited responsiveness, ${ }^{46}$ multiple sclerosis clinical trialists are looking for more rigorous 
methods of measurement of health status. There is, rightly, considerable interest in the SF-36. Furthermore, the benefits of SF-36 summary scores over scale scores (absence of floor and ceiling effects, better measurement precision, greater responsiveness, fewer statistical analyses) offer an attractive solution to many measurement problems. Results from this and other studies ${ }^{15} 22$ show floor and ceiling effects for SF-36 scales that may limit their usefulness in detecting treatment effects, and this study questions the validity of SF-36 summary scores.

This study also has wider implications for health measurement. The findings are of practical use for neurologists and other health professionals who may take measures "off the shelf" expecting that such attributes have been fully tested or that measures are generally applicable. The result might also be important in reporting accurately the strength of treatment or rehabilitation effects.

\section{Conclusions}

The findings of this study provide some empirically based guidelines for the use of the SF-36 in multiple sclerosis. High levels of data quality support its use as a health measure in diverse groups of people with multiple sclerosis. Tests of scaling assumptions suggest that SF-36 scale scores can be reported with confidence, but that summary scores are not valid. Results also suggest that use of the SF-36 might be limited to cross sectional studies as the floor and ceiling effects associated with scale scores could underestimate treatment effectiveness in clinical trials, and the extent of health changes in longitudinal studies. It is important to note that health measures such as the SF-36 are recommended for group comparison studies and not individual patient clinical decision making. This is because confidence intervals around individual scores are too wide to be able to make reliable and valid judgments at the level of the individual patient. ${ }^{39}$ Finally, this study provides further evidence of the need to evaluate psychometric properties, including scaling assumptions, in relevant clinical populations before these instruments are used to evaluate therapeutic effectiveness in disease specific samples.

We are very grateful to the people with multiple sclerosis who participated in the study, the multiple sclerosis Society of Great Britain and Northern Ireland for their collaboration in this project, and Ms Laura Camfield at the Institute of Neurology for help with the postal survey.

1 McHorney CA, Ware JE Jr, Lu JFR, et al. The MOS 36-item short-form health survey (SF-36): III. Tests of data quality, scaling assumptions and reliability across diverse patient groups. Med Care 1994;32:40-66

2 Kosinski M, Keller SD, Hatoum TH, et al. The SF-36 health survey as a generic outcome measure in clinical trials of patients with rheumatiod arthritis. Tests of data quality, scaling assumptions, and score reliability. Med Care 1999;37:MS10-22.

3 Ware JE Jr, Snow KK, Kosinski M, et al. SF-36 health survey manual and interpretation guide. Boston, MA: Nimrod Press, 1993.

4 Stewart AL, Hays RD, Ware JE Jr. The MOS short-form general health survey: reliability and validity in a patient population. Med Care 1988;26:724-35.

5 Dorman P, Slattery J, Farrell B, et al. Qualitative comparison of the reliability of health status assessments with EuroOol and SF-36 questionnaires after stroke. Stroke 1998;29:63-8.
6 Jenkinson C, Fitzpatrick R, Swash M, et al. The ALS health profile study: quality of life of ALS patients and carers in europe. F Neurol 2000;247:0835-40.

7 Jenkinson C, Peto V, Fitzpatrick R, et al. Self-reported functioning and well-being in patients with Parkinson's disease: comparison of the short-form health survey (SF-36) and the Parkinson's disease questionnaire (PDQ-39). Age Ageing 1995;24:505-9.

8 Jacoby A, Baker G, Steen N, et al. The SF-36 as a health status measure for epilepsy: a psychometric analysis. Qual Life Res 1999;8:351-64.

9 Monzon M, Lainesz M. Quality of life in migraine and chronic daily headache patients. Cephalagia 1998;18:63843.

10 Freeman JA, Langdon DW, Hobart JC, et al. Health-related quality of life in people with multiple sclerosis undergoing

Rothwell PM, McDowell Z Wong CK, et al. Doctors and patients don't agree: cross sectional study of patients' and patients don't agree: cross sectional study of patients' and ple sclerosis. BMF 1997;314:1580-3.

12 The Canadian Burden of Illness Study Group. Burden of illness of multiple sclerosis: part II: quality of life. Can $\mathcal{F}$ Neurol Sci 1998;25:31-8.

13 Vickrey BG, Hays RD, Genovese BJ, et al. Comparison of generic to disease-targeted health-related quality-of-life measures for multiple sclerosis. F Clin Epidemiol 1997;50: $557-69$.

14 Peto V, Jenkinson C, Fitzpatrick R, et al. The development and validation of a short measure of functioning and wellbeing for individuals with Parkinson's disease. Qual Life Res $1995 ; 4: 241-8$

15 Vickrey BG, Hays RD, Harooni R, et al. A health-related quality of life measure for multiple sclerosis. Qual Life Res 1995;4:187-206.

16 Cella DF, Dineen K, Arnason B, et al. Validation of the functional assessment of multiple sclerosis quality of life instrument. Neurology 1996;47:129-39.

17 Jenkinson C, Fitzpatrick R, Brennan C, et al. Development and validation of a short measure of health status for individuals with ALS/MND. F Neurol 1999;246:16-21.

18 Schrag A, Selai C, Jahanshahi M, et al. The EQ-5D-a generic quality of life measure-is a useful instrument to measure quality of life in patients with Parkinson's disease. 7 Neurol Neurosurg Psychiatry 2000;69:67-73.

19 Martin B, Pathak D, Sharfman M, et al. Validity and reliability of the migraine-specific quality of life questionnaire (MSQ version 2.1). Headache 2000;40:204-15.

20 Ware JE Jr, Kosinski MA, Keller SD. SF-36 physical and mental health summary scales: a user's manual. Boston, MA: The Health Institute, New England Medical Centre, 1994.

21 Ware JE Jr, Kosinski M, Bayliss MS, et al. Comparison of methods for the scoring and statistical analysis of SF-36 health profile and summary measures: summary of results from the medical outcomes study. Med Care 1995;33: AS264-79.

22 Vickrey B, Hays R, Genovese B, et al. Comparison of a generic to disease-targeted health-related quality-of-life measures for multiple sclerosis. $\mathcal{F}$ Clin Epidemiol 1997;50: $557-69$.

23 Freeman JA, Hobart JC, Langdon DW, et al. Clinical appropriateness: a key factor in outcome measure selection. The 36-item short form health survey in multiple sclerosis. F Neurol Neurosurg Psychiatry 2000;68:150-6.

24 Lord FM, Novick MR. Statistical theories of mental test scores. Reading, MA: Addison-Wesley, 1968.

25 Poser CM, Paty DW, McDonald WI, et al. New diagnostic criteria for multiple sclerosis: guidelines for research protocols. Ann Neurol 1983;13:227-31.

26 Kurtzke JF. Rating neurological impairment in multiple sclerosis: an expanded disability status scale (EDSS). Neurology 1983;33:1444-52.

27 Hobart JC, Lamping DL, Fitzpatrick R, et al. The multiple sclerosis impact scale (MSIS-29): a new patient-based outcome measure. Brain 2001;124:962-73.

28 Likert RA. A technique for the development of attitudes. Arch Psychol 1932;140:5-55.

29 Stewart AL, Ware JE Jr, ed. Measuring functioning and well-being: the medical outcomes study approach. Durham, NC: Duke University Press; 1992

30 Ware JE Jr, Davies-Avery A, Brook RH. Conceptualization and measurement of health for adults in the health insurance study. Vol VI. Analysis of relationships among health status measures. Santa Monica, CA: The Rand Corporation, 1980. (Report No R-1987/6-HEW.)

31 Streiner DL, Norman GR. Health measurement scales: a practical guide to their development and use, 2 nd ed. Oxford: Oxford University Press, 1995.

32 Nunnally JC, Bernstein IH. Psychometric theory, 3rd ed. New York: McGraw-Hill, 1994.

33 Ware JE Jr, Harris WJ, Gandek B, et al. $M A P-R$ for windows: multitrait/multi-item analysis program: revised user's guide. Boston, MA: Health Assessment Lab, 1997.

34 Ware JE Jr, Brook RH, Davies-Avery A, et al. Conceptualization and measurement of health for adults in the health insurance study. Vol I. Model of health and methodology. Santa Monica, California: The Rand Corporation, 1980. (Report No R-1987/1-HEW.

35 Cronbach LJ. Coefficient alpha and the internal structure of tests. Psychometrika 1951;16:297-334. 
36 Lohr KN, Aaronson NK, Alonso J, et al. Evaluating quality of life and health status instruments: development of scientific review criteria. Clin Ther 1996;18:979-92.

37 Guttman LA. Some necessary conditions for commonfactor analysis. Psychometrika 1954;19:149-61.

38 Cattell RB. The scree test for the number of factors. Multivariate Behavioural Research 1966;1:245-76.

39 McHorney CA, Tarlov AR. Individual-patient monitoring in clinical practice: are available health status surveys adequate? Qual Life Res 1995;4:293-307.

40 Brazier JE, Harper R, Jones NMB, et al. Validating the SF 36 health survey questionnaire: new outcome measure for primary care. $B M \mathcal{F} 1992 ; 305: 160-4$.

41 Simon GE, Revicki DA, Grothaus MA, et al. SF-36 summary scores: are physical and mental health truly distinct? Med Care 1998;36:567-72.
42 Hays RD, Prince-Embury S, Chen H. RAND-36 Health status inventory. San Antonio, TX: Psycholoical Corporastatus inventor
tion, 1998 .

43 Norvedt MW, Riise T, Myer K-M, et al. Performance of the SF-36, SF-12, and RAND-36 summary scales in a multiple sclerosis population. Med Care 2000;38:1022-8.

44 Fayers PM, Machin D. Factor analysis. In: Staquet MJ, Hays RD, Fayers PM, eds. Quality of life assessment in clinical trials: methods and practice. Oxford: Oxford University Press, 1998:191-223.

45 Jenkinson C. Comparison of UK and US methods for weighting and scoring the SF-36 summary measures. $\mathcal{F}$ Public Health Med 1999;21:372-6.

46 Hobart JC, Freeman JA, Thompson AJ. Kurtzke scales revisited: the application of psychometric methods to clinical intuition. Brain 2000;123:1027-40.

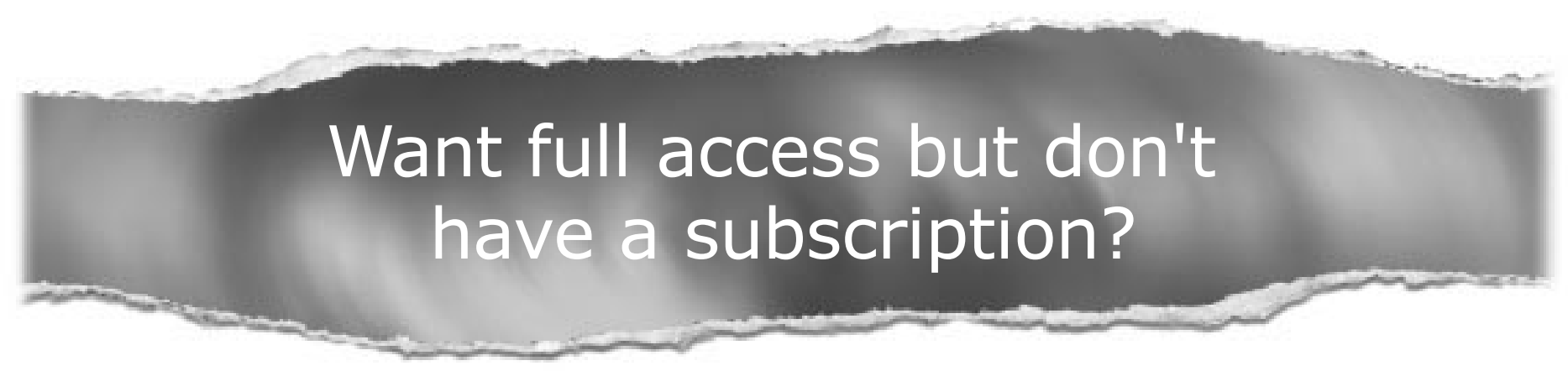

\section{Pay per access}

For just US $\$ 25$ you can have instant access to the whole website for 30 days. During this time you will be able to access the full text for all issues (including supplements) available. You will also be able to download and print any relevant pdf files for personal use, and take advantage of all the special features Journal of Neurology, Neurosurgery, and Psychiatry online has to offer.

\section{www.jnnp.com}

